\title{
Extended follow-up of a short total diet replacement programme: results of the Doctor Referral of Overweight People to Low Energy total diet replacement Treatment (DROPLET) randomised controlled trial at 3 years
}

\author{
Nerys M. Astbury $\mathbb{D}^{1 凶}$, Rhiannon M. Edwards ${ }^{1}$, Fitsum Ghebretinsea ${ }^{1}$, Milensu Shanyinde ${ }^{1}$, Jill Mollison ${ }^{1}$, Paul Aveyard $\mathbb{D}^{1}$ and $^{\circ}$ \\ Susan A. Jebb ${ }^{1}$
}

(c) The Author(s) 2021

\begin{abstract}
OBJECTIVES: To test the long-term effectiveness of a total diet replacement programme (TDR) for routine treatment of obesity in a primary care setting.

METHODS: This study was a pragmatic, two-arm, parallel-group, open-label, individually randomised controlled trial in adults with obesity. The outcomes were change in weight and biomarkers of diabetes and cardiovascular disease risk from baseline to 3 years, analysed as intention-to-treat with mixed effects models.

INTERVENTIONS: The intervention was TDR for 8 weeks, followed by food-reintroduction over 4 weeks. Behavioural support was provided weekly for 8 weeks, bi-weekly for the next 4 weeks, then monthly for 3 months after which no further support was provided. The usual care (UC) group received dietary advice and behavioural support from a practice nurse for up to 3 months. RESULTS: Outcome measures were collected from 179 (66\%) participants. Compared with baseline, at 3 years the TDR group lost $-6.2 \mathrm{~kg}$ (SD 9.1) and usual care $-2.7 \mathrm{~kg}$ (SD 7.7); adjusted mean difference $-3.3 \mathrm{~kg}$ (95\% $\mathrm{Cl}:-5.2,-1.5), p<0.0001$. Regain from programme end ( 6 months) to 3 years was greater in TDR group $+8.9 \mathrm{~kg}$ (SD 9.4) than UC +1.2 , (SD 9.1); adjusted mean difference $+6.9 \mathrm{~kg}(95 \% \mathrm{Cl} 4.2,9.5) P<0.001$. At 3 years TDR led to greater reductions than UC in diastolic blood pressure (mean difference $-3.3 \mathrm{mmHg}(95 \% \mathrm{Cl}:-6.2 ;-0.4) P=0.024)$, and systolic blood pressure (mean differences $-3.7 \mathrm{mmHg}(95 \% \mathrm{Cl}:-7.4 ; 0.1) P=$ 0.057). There was no evidence of differences between groups in the change from baseline to 3 years $\mathrm{HbA} \mathrm{A}_{1 \mathrm{c}}(-1.9 \mathrm{mmol} / \mathrm{mol}(95 \%$ $\mathrm{Cl}:-0.7 ; 4.5 ; P=0.15), \mathrm{LDL}$ cholesterol concentrations $(0.2 \mathrm{mmol} / \mathrm{L}(95 \% \mathrm{Cl}-0.3,0.7) P=0.39)$, cardiovascular risk score (QRISK2) $(-0.37(95 \% \mathrm{Cl}-0.96 ; 0.22) ; P=0.22)$.

CONCLUSIONS: Treatment of people with obesity with a TDR programme compared with support from a practice nurse leads to greater weight loss which persists to at least 3 years, but there was only evidence of sustained improvements in BP and not in other aspects of cardiometabolic risk.
\end{abstract}

International Journal of Obesity (2021) 45:2432-2438; https://doi.org/10.1038/s41366-021-00915-1

\section{INTRODUCTION}

The results from several international trials investigating the clinical effectiveness of programmes that combine low-energy total diet replacement (TDR) with behavioural support have consistently shown that these TDR programmes are an effective treatment for obesity [1-6]. They promote rapid weight loss over 12-16 weeks and lead to significant and substantial weight loss at 1 year compared with behavioural weight-loss programmes which do not include meal replacements. There is growing interest from healthcare commissioners and policymakers for the potential of TDR as a routine treatment for obesity. The National Health Service (NHS) in England has announced a pilot rollout of this treatment, involving referral of 5000 patients with type 2 diabetes $[7,8]$ to a 12 week TDR weight loss programme. However, evidence on longer-term outcomes is limited [9].

Unlike most other recent TDR trials $[2,3,6,10]$, the DROPLET study tested the effectiveness of a TDR for routine treatment of obesity, rather than as a specific treatment aimed at diabetes remission. In the DROPLET trial, at 1 year participants in the TDR group lost over $10 \mathrm{~kg}, 7 \mathrm{~kg}$ more than those receiving usual care (UC), with $45 \%$ of participants in the TDR group achieving a weight loss of $10 \%$ or more [1]. Diastolic blood pressure was $3.1 \mathrm{mmHg}$ lower, with $\mathrm{HbA} 1 \mathrm{c}$ reductions of $2.2 \mathrm{mmol} / \mathrm{mol}$ compared with UC. The intervention was delivered by referring participants to existing

${ }^{1}$ Nuffield Department of Primary Care Health Sciences, University of Oxford, Oxford, UK. ${ }^{凶}$ email: nerys.astbury@phc.ox.ac.uk 


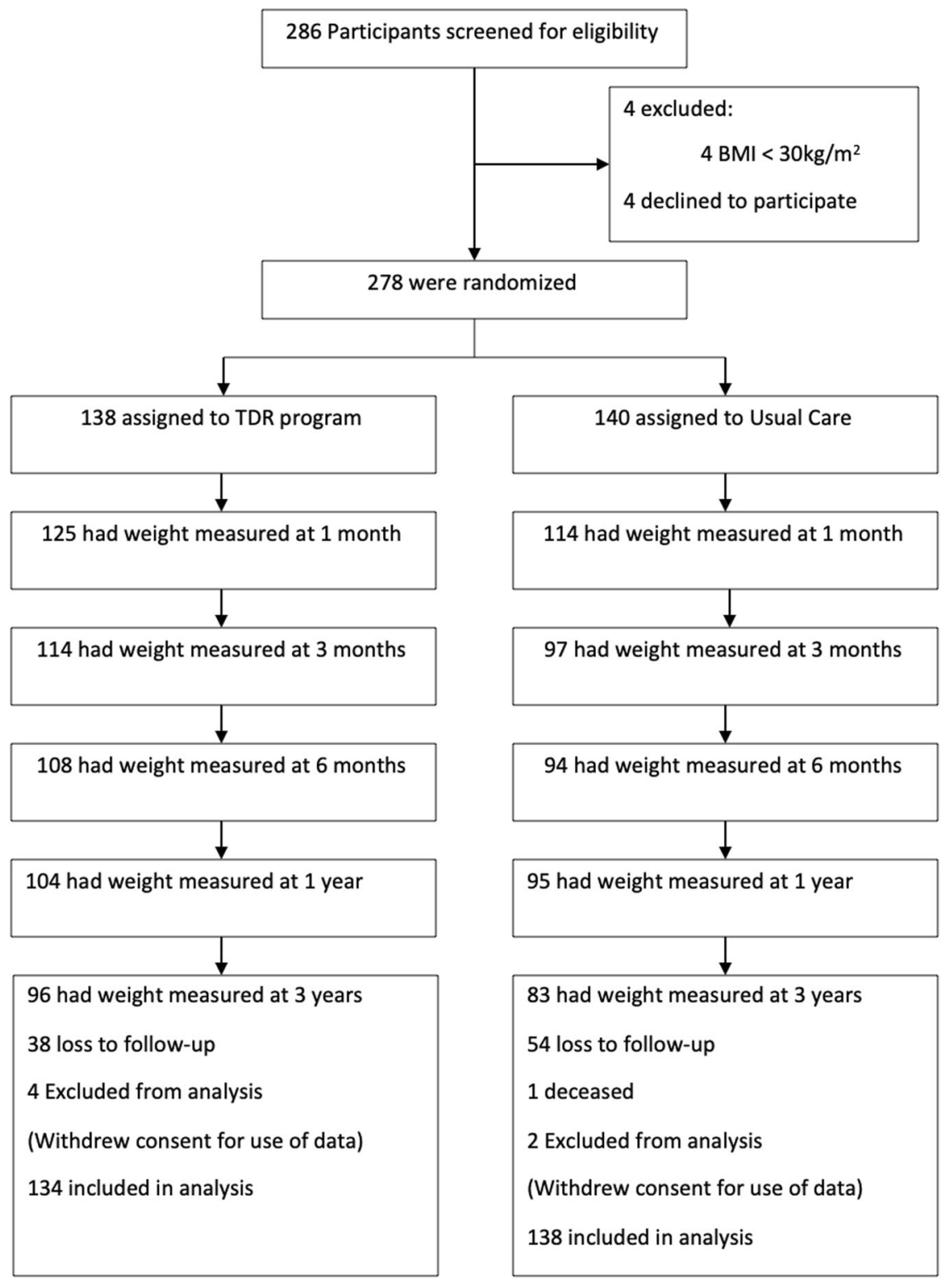

Fig. 1 Consort flowchart.

counsellors in the community and economic modelling suggesting that this intervention delivery model was a cost-effective option for treating adults with obesity [11]. This analysis assumed that weight was fully regained over 5 years and that cardiometabolic risk followed a similar pattern. However, this was based on assumptions about weight regain from trials of other weight loss interventions and there is concern that weight regain may be quicker after TDR programmes than other programmes associated with a slower rate of weight loss or those that do not rely on special products $[11,12]$.

The aim of this study was to determine longer-term weight and health outcomes of the participants of the DROPLET randomised controlled trial. These data will allow healthcare professionals to determine the clinical value of TDR programmes, help in assessing cost-effectiveness, and inform healthcare commissioners.

\section{METHODS}

The DROPLET trial was a pragmatic, individually randomised, two-arm, open-label, parallel trial. The participants were randomised in a 1:1 allocation stratified for BMI $\left(<35 \mathrm{kgm}^{2}\right.$ or $\left.\geq 35 \mathrm{kgm}^{2}\right)$ and type 2 diabetes status to either a TDR treatment or UC (control). A full protocol for the original trial has been published previously [13]. This study was powered to detect a difference in body weight between groups at 1 year (primary outcome) [1]. We obtained additional ethical approval to conduct a 3-year follow-up for this study from NHS NRES South Central Oxford B Research Ethics committee (Ref: 19/SC/0012) and the follow up of the trial was registered with ISRCTN12311645. All participants gave written informed consent before enrolment to the follow-up study.

\section{Participants and settings}

Eligible participants included all those who had taken part in the DROPLET trial, except the few participants who withdrew prior to the 1-year followup (Fig. 1). Full trial inclusion and exclusion criteria have been reported previously, but we enrolled adults with obesity, with few exclusions [1, 13]. Eligible participants were re-contacted around the time of the 3 year anniversary of their enrolment on to the DROPLET trial. They were invited to attend an appointment at their GP practice to measure body weight, blood pressure and to provide a fasting blood sample. Participants who were unable to attend follow-up in person were asked if we could use a recent weight and health measures from their medical record for this study. The researchers extracted this information from the participants records if it was available. Where it was not available, participants were 
asked to provide a self-reported weight measurement. All participants were asked to complete a questionnaire measuring quality of life. Three year follow-ups took place between March 2019 and September 2019.

\section{Interventions}

The TDR programme was provided by Cambridge Weight Plan UK, which manages a network of counsellors providing behavioural support and food products. Participants were asked to contact a local counsellor who delivered a 24-week TDR weight loss programme consisting of 8 weeks TDR, 4 weeks gradual food-reintroduction, and a further 12 weeks weight maintenance. Behavioural support was provided weekly for 8 weeks, biweekly for 4 weeks, and monthly for 3 months.

For participants randomised to the UC comparator, practice nurses offered a weight loss programme for 12 weeks, at a frequency typically used in the practice (e.g. weekly or bi-weekly). Full details of the interventions provided as part of the trial have been published previously [13].

Consistent with the pragmatic design, participants in both groups were free to use other weight loss programmes or products during and after their assigned interventions, but GPs were asked not to refer participants to other weight loss programmes for the first 12 months.

\section{Procedures}

At baseline and follow-up, weight and body fat were measured on a digital scale (TANITA SC-240). Waist circumference was measured at the top of the iliac crest [14]. Seated blood pressure was measured three times using an automated blood pressure monitor, with the mean of the last two measurements used for analysis. Measurements were collected by research nurses or a trained research assistant. A fasting blood sample was collected and analysed for glucose, insulin, triglycerides and cholesterol fractions. Participants were asked to complete questionnaires booklet, which recorded quality of life using the EQ5D and obesity-specific OWL-QOL [15-17].

\section{Outcomes}

The primary outcome was change in body weight from baseline to 3 years. The secondary outcomes were: the proportion of participants achieving $5 \%$ and $10 \%$ weight loss, change in fat mass, LDL cholesterol, $\mathrm{HbA1c}$, systolic and diastolic blood pressure, and 10-year cardiovascular risk using QRISK2 between baseline and 3 years. Although participants were not blinded to treatment allocation, the research nurses and researchers who collected the outcome measures for this 3 year follow-up were blinded to the participants treatment allocation.

Pre-specified exploratory outcomes were change from baseline to 3 years in waist circumference, fasting triglycerides, $\mathrm{HDL}$ cholesterol, glucose and insulin, as well as derived HOMA-IR, HOMA-\% $\beta$, and HOMA-\%S calculated using the HOMA2 calculator, and changes EQ-5D and OWL-QOL [15-17]. We also examined weight change from 6 months (end of TDR programme) to 3 years.

\section{Statistical analysis}

We followed a statistical analysis plan finalised prior to database lock. The primary, secondary and exploratory outcomes were all analysed by an independent trial statistician following an intention-to-treat (ITT) analysis in Stata Version 14.0. The primary outcome was analysed using a linear mixed effect model with randomised group, visit, the interaction between visit and randomised group, baseline weight and baseline variables predictive of missing primary outcome (age, gender and diabetes status) included as fixed effects. Practice and participant were included as random effects in the model, to account for repeated measures on the same participant at 3 months, 6 months and 1 and 3 years. An unstructured variance covariance matrix was specified. The analysis of secondary and exploratory outcomes followed the same strategy as the primary outcome with baseline BMI and baseline outcome (if applicable) substituted for baseline weight as a fixed effects. Weight loss of $\leq 5 \%$ and $\leq 10 \%$ at 3 years was analysed using a mixed effect logistic regression model.

We assessed the sensitivity of the primary outcome measure to missing data using different imputation methods; baseline and last observation carried forward, completers only, multiple imputation, and a pattern mixture model assuming different degrees of missing not at random.

To assess whether treatment effects differed by age, gender, BMI, diabetes status and socioeconomic deprivation we performed prespecified exploratory subgroup analyses.
Participants home postcode were used to calculate patients' socioeconomic deprivation using the Index of Multiple Deprivation (IMD) [18]. The IMD ranks geographical areas of about 500 households in the UK on seven indices: income, employment, health deprivation and disability, education, crime, barriers to housing and services, and living environment. These ranks are grouped into deciles which were used for analysis with lowest decile [1] representing the most deprived areas and highest decile [10] representing the least deprived areas.

\section{Patient involvement}

All participants in the original trial were sent a summary of the main results and link to access the full manuscript when the results were published in September 2018. Feedback from participants was positive overall, with many commenting that the magnitude of weight loss achieved by the TDR programme was impressive. However, several participants raised concerns regarding the sustainability of the effects. This feedback was integral to our decision to seek support to measure the effects.

\section{RESULTS}

Six of the 278 randomised participants withdrew consent for their data to be used after randomisation, leaving 272 participants eligible for follow-up. At the 3 year follow up we collected outcome data from 179 (66\%) participants; 96 (72\%) from the TDR group and 83 (62\%) from UC group respectively. Body weight was objectively measured in $90(94 \%)$ and 78 (94\%) participants in TDR and UC respectively, with the remaining $11(6 \%)$ of weight outcomes based on self-reported measures (Fig. 1)

Most participants were middle aged, $55 \%$ were women, and $90 \%$ were white British. The mean baseline BMl was $36.8 \mathrm{~kg} / \mathrm{m}^{2}$, $20 \%$ were diagnosed with type 2 diabetes and $25 \%$ had hypertension at baseline (Table 1).

\section{Primary outcome}

Weight change at 3 years was $-6.3 \mathrm{~kg}$ (SD 9.1) in the TDR group and $-2.7 \mathrm{~kg}$ (7.7) in the control group (Fig. 2), adjusted difference $-3.3 \mathrm{~kg}(-9.4 ;-4.9), p<0.0001$. Sensitivity analyses for different assumptions about loss to follow-up did not change the findings (Table S1). In addition sensitivity analysis excluding self-reported outcomes did not change the findings (Table S2).

There was no evidence that the effect of the intervention on weight change differed by age group ( $p=0.37$ ), baseline BMI ( $p=$ 0.73 ) or diabetes status $(p=0.35)$. However, the mean difference between TDR and control groups for weight change from baseline to 3 years was less in women than in men $(p=0.039)$, and there was an indication that participants from more deprived neighbourhoods also received less benefit from TDR than participants from less deprived areas $(p=0.058)$ (Fig. S1).

\section{Secondary outcomes}

At 3 years, $46 \%$ and $35 \%$ of participants in the TDR and UC group respectively who attended follow-up had lost $\geq 5 \%$ of their baseline weight, odds ratio (OR) $1.5(0.8 ; 2.8), p=0.20$. Twentyfour percent and $13 \%$ of participants lost $\geq 10 \%$ baseline weight in the TDR and UC groups respectively; OR $1.9(0.8 ; 4.2), p=0.13$ (Fig. S2). The change in fat mass showed similar changes to total body weight, albeit smaller (Table 2).

At 3 years, there was no evidence of differences between groups in change in $\mathrm{HbA}_{1 c}$ or LDL cholesterol. Both systolic and diastolic blood pressure were lower in the TDR group although the between group difference was not significant for systolic blood pressure (Table 2). There was no evidence of a difference in the change in QRISK2 score between groups.

In a post-hoc analysis, we examined the effectiveness of the TDR programme for people with diabetes or hypertension (Table S3 and S4). We advocated reductions in medication for hypertension or diabetes in the initial phase of the TDR programme, which may have masked changes in blood pressure and glucose 
Table 1. Baseline characteristics ${ }^{\mathrm{a}}$.

\begin{tabular}{|c|c|c|c|}
\hline & $N$ & Usual care & $\begin{array}{l}\text { Total diet } \\
\text { replacement }\end{array}$ \\
\hline Age (yrs) & 179 & $50.9 \pm 11.7$ & $50.7 \pm 11.2$ \\
\hline \multicolumn{4}{|l|}{ Gender n (\%) } \\
\hline Female & 99 & $45(54.2)$ & $54(56.3)$ \\
\hline Male & 80 & $38(45.8)$ & $42(43.8)$ \\
\hline \multicolumn{4}{|l|}{ Ethnicity } \\
\hline White British & 162 & $75(86.2)$ & $87(90.6)$ \\
\hline Not White British & 17 & $4(23.5)$ & $9(52.9)$ \\
\hline IMD decile ${ }^{b}$ & 179 & $7.3 \pm 2.0$ & $7.6 \pm 2.0$ \\
\hline Weight (kg) & 179 & $105.6 \pm 18.8$ & $107.4 \pm 18.8$ \\
\hline Height (cm) & 179 & $169.0 \pm 9.6$ & $169.2 \pm 9.3$ \\
\hline BMI $\left(\mathrm{kg} / \mathrm{m}^{2}\right)$ & 179 & $36.1 \pm 4.3$ & $37.5 \pm 6.0$ \\
\hline Waist circumference $(\mathrm{cm})$ & 177 & $114.0 \pm 10.5$ & $116.5 \pm 13.5$ \\
\hline Body fat (\%) & 172 & $41.2 \pm 7.3$ & $42.5 \pm 8.1$ \\
\hline \multicolumn{4}{|l|}{ Blood pressure $(\mathrm{mmHg})$} \\
\hline Systolic & 176 & $130.9 \pm 15.2$ & $132.6 \pm 15.7$ \\
\hline Diastolic & 176 & $80.8 \pm 9.9$ & $83.8 \pm 9.3$ \\
\hline \multicolumn{4}{|l|}{ Medical conditions $n(\%)$} \\
\hline Type 2 diabetes & 179 & $20(14.5)$ & $21(15.7)$ \\
\hline Hypertension & 179 & $30(21.7)$ & $33(24.6)$ \\
\hline $\mathrm{HbA} 1 \mathrm{c}(\mathrm{mmol} / \mathrm{mol})$ & 176 & $40.2 \pm 12.6$ & $40.7 \pm 12.8$ \\
\hline $\begin{array}{l}\text { Fasting blood glucose } \\
(\mathrm{mmol} / \mathrm{L})\end{array}$ & 175 & $5.8 \pm 2.1$ & $6.1 \pm 2.7$ \\
\hline Fasting insulin (pmol/L) & 174 & $105.3 \pm 94.4$ & $95.8 \pm 50.6$ \\
\hline HOMA -IR & 171 & $1.9 \pm 1.5$ & $1.9 \pm 1.3$ \\
\hline HOMA $\beta$ (\%) & 171 & $129.2 \pm 91.6$ & $115.0 \pm 56.3$ \\
\hline HOMA S (\%) & 171 & $69.0 \pm 32.1$ & $69.3 \pm 36.2$ \\
\hline \multicolumn{4}{|l|}{ Cholesterol (mmol/L) } \\
\hline Total & 178 & $5.0 \pm 1.1$ & $5.1 \pm 1.1$ \\
\hline $\begin{array}{l}\text { High-density } \\
\text { lipoprotein }\end{array}$ & 178 & $1.2 \pm 0.3$ & $1.2 \pm 0.3$ \\
\hline $\begin{array}{l}\text { Low-density } \\
\text { lipoprotein }\end{array}$ & 172 & $3.1 \pm 1.0$ & $3.2 \pm 0.9$ \\
\hline Triglycerides (mmol/L) & 178 & $1.7 \pm 0.8$ & $1.7 \pm 0.9$ \\
\hline QRISK2 (\%) & 175 & $9.6 \pm 9.0$ & $9.5 \pm 8.7$ \\
\hline
\end{tabular}

${ }^{a}$ Values represent means \pm SD or proportion.

bIndex of Multiple Deprivation (IMD) ranks geographical areas of about 500 households in the UK on seven indices: income, employment, health deprivation and disability, education, crime, barriers to housing and services and living environment. These ranks are grouped into deciles which were used for analysis with lowest decile representing the most deprived areas and highest decile representing the least deprived area.

regulation. In these small subgroups there was no evidence that medication changes differed significantly between groups.

\section{Exploratory outcomes}

Changes between end of programme ( 6 months) and 3 years. Both groups gained weight between 6 months (the end of the programme) and 3 years. The control group gained $1.2 \mathrm{~kg}$ (SD 9.1) and the intervention group gained $8.9 \mathrm{~kg}$ (SD 9.4), adjusted difference $+6.9 \mathrm{~kg}(95 \% \mathrm{Cl} 4.2,9.5)$.

The change in waist circumference showed a similar pattern to changes in body weight and fat mass. There was no evidence of differences between groups in glucose regulation, triglycerides or cholesterol fractions between 6 months and 3 years (Table 2).

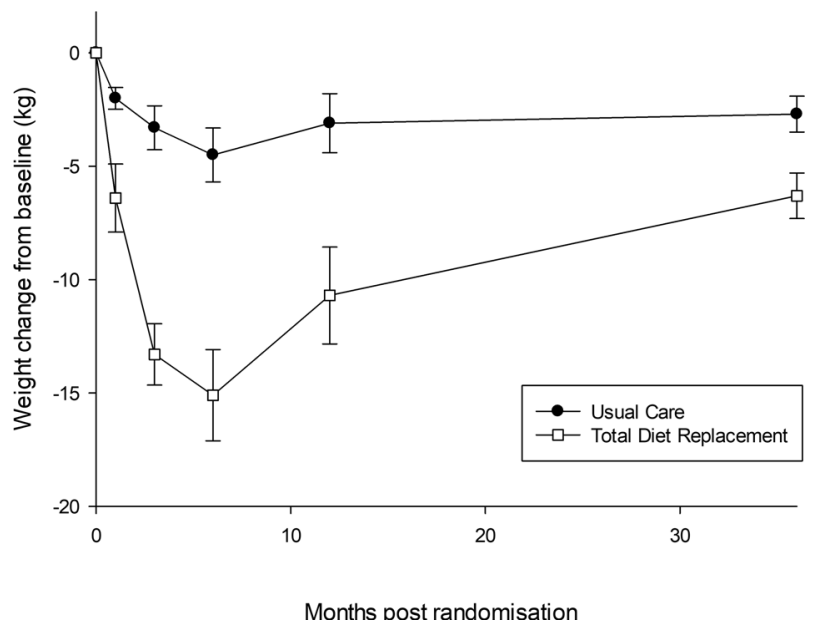

Fig. 2 Weight change over 36 months in the intention-to-treat population*. *values represent means \pm SEM.

Quality of life. Quality of life measures at 3 years improved in both groups compared with baseline with no evidence of a difference between groups except for the visual analogue scale component of EQ-5D, where the improvement was greater in the UC group (Table 2).

Self-reported weight loss attempts. At 3 years, 70\% participants in the TDR group and 68\% in the UC group who attended follow up reported attempting to lose weight in the 4 weeks prior to their appointment. Of those reporting trying to lose weight, $49 \%$ of people in the TDR group, and $50 \%$ in the UC group reported that they were attending a formal weight management programme. The mean weight loss at 3 years of the participants reporting trying to lose weight compared with those not currently trying to lose weight was $-7.6 \mathrm{~kg}$ versus $-4.8 \mathrm{~kg}$ in the TDR group and $-3.6 \mathrm{~kg}$ versus $-1.6 \mathrm{~kg}$ in the UC group.

\section{DISCUSSION}

Participants randomised to a TDR and behavioural support programme for weight loss were on average $6 \mathrm{~kg}$ lighter than at baseline, and lost $3 \mathrm{~kg}$ more than participants randomised to a UC weight loss programme after 3 years. This is despite greater regain from the end of the programme (weight loss nadir) to 3 years in the TDR group $(+8.9 \mathrm{~kg})$ compared with the control $(+1.2 \mathrm{~kg})$. Blood pressure was somewhat lower in the TDR group compared with UC, albeit the difference in systolic pressure difference was not significant. There was no clear pattern and no significant differences in measures of glucose regulation. Lipid changes were likewise not significantly different between the groups. Although some people with hypertension and diabetes reduced medication, there is no evidence this accounted for the lack of observed effect of the intervention on cardiovascular risk. Both the main EQ-5D factor score and OWLQOL scales showed improvements in quality of life from baseline but no evidence of differences between groups, although the EQ-5D visual analogue scale showed a significantly greater improvement in quality of life in the UC over the TDR group.

\section{Strengths and limitations}

The major strength of this study is the pragmatic design, which can provide an estimate of the likely outcomes from routine referral systems for the treatment of obesity. Limitations include that the trial was originally planned to assess outcomes at 1 year only. An amendment to the protocol was made to include a follow-up at 3 years, but this was a post-hoc decision after seeing 
Table 2. Primary, secondary and exploratory outcomes by group.

\begin{tabular}{|c|c|c|c|c|c|c|}
\hline \multirow[b]{3}{*}{3 years } & \multicolumn{4}{|c|}{ Change from baseline (mean \pm SD) } & \multicolumn{2}{|l|}{ Treatment difference } \\
\hline & Total diet replacement & $n$ & Usual care & $n$ & Adjusted difference $(95 \% \mathrm{CI})$ & $p$ value \\
\hline & & & & & & \\
\hline Weight $(\mathrm{kg})^{\mathrm{a}}$ & $-6.3 \pm 9.1$ & 96 & $-2.7 \pm 7.7$ & 83 & $-3.3(-5.2,-1.5)$ & $<0.001$ \\
\hline Lost $\geq 5 \%$ weight $n(\%)^{b}$ & $44(45.8)$ & 96 & $29(34.9)$ & 83 & OR; $1.5(0.8,2.8)$ & 0.202 \\
\hline Lost at least $\geq 10 \%$ weight $n(\%)^{b}$ & $23(24.0)$ & 96 & $11(13.3)$ & 83 & OR; $1.9(0.8,4.2)$ & 0.126 \\
\hline Fat mass $(\mathrm{kg})^{\mathrm{b}}$ & $-4.9 \pm 10.1$ & 76 & $-1.9 \pm 6.7$ & 69 & $-2.4(-4.4 ;-0.5)$ & 0.016 \\
\hline Diastolic Blood Pressure $(\mathrm{mmHg})^{b}$ & $-1.2 \pm 9.9$ & 77 & $3.8 \pm 10.9$ & 68 & $-3.3(-6.2 ;-0.4)$ & 0.024 \\
\hline $\mathrm{HbA} 1 \mathrm{c}(\mathrm{mmol} / \mathrm{mol})^{\mathrm{b}}$ & $3.1 \pm 9.8$ & 71 & $-0.8 \pm 6.4$ & 61 & $1.9(-0.7 ; 4.5)$ & 0.152 \\
\hline QRISK2 (\%) ${ }^{\mathrm{b}}$ & $0.98 \pm 2.77$ & 57 & $1.46 \pm 2.15$ & 50 & $-0.37(-0.96 ; 0.22)$ & 0.222 \\
\hline Waist circumference $(\mathrm{cm})^{c}$ & $-10.5 \pm 9.1$ & 77 & $-5.5 \pm 7.3$ & 70 & $-3.5(-5.7 ;-1.4)$ & 0.001 \\
\hline Fasting glucose $(\mathrm{mmol} / \mathrm{L})^{c}$ & $-0.5 \pm 1.8$ & 59 & $0.1 \pm 1.3$ & 47 & $-0.4(-0.8 ;-0.1)$ & 0.396 \\
\hline Total Cholesterol $(\mathrm{mmol} / \mathrm{L})^{c}$ & $-0.1 \pm 0.8$ & 69 & $-0.2 \pm 0.9$ & 61 & $0.2(-0.1,0.4)$ & 0.264 \\
\hline $\mathrm{HDL}$ cholesterol $(\mathrm{mmol} / \mathrm{L})^{\mathrm{c}}$ & $0.1 \pm 0.3$ & 69 & $0.1 \pm 0.2$ & 61 & $0.00(-0.1 ; 0.1)$ & 0.969 \\
\hline LDL Cholesterol $(\mathrm{mmol} / \mathrm{L})^{\mathrm{b}}$ & $-0.1 \pm 0.7$ & 61 & $-0.2 \pm 0.7$ & 56 & $0.1(-0.10 ; 0.34)$ & 0.295 \\
\hline Triglycerides $(\mathrm{mmol} / \mathrm{L})^{\mathrm{c}}$ & $-0.2 \pm 0.6$ & 63 & $-0.1 \pm 0.9$ & 58 & $-0.1(-0.4 ;-0.1)$ & 0.394 \\
\hline \multicolumn{7}{|l|}{ Quality of Life: } \\
\hline EQ-5D (Index) ${ }^{c}$ & $0.00 \pm 0.17$ & 67 & $0.02 \pm 0.016$ & 70 & $-0.02(-0.07 ; 0.03)$ & 0.419 \\
\hline EQ-5D (VAS) ${ }^{c}$ & $6.6 \pm 19.9$ & 79 & $22.6 \pm 115.1$ & 70 & $-18.0(32.2 ; 3.8)$ & 0.013 \\
\hline $\mathrm{OWL}-\mathrm{QOL}{ }^{\mathrm{c}}$ & $9.5 \pm 16.2$ & 77 & $8.1 \pm 14.7$ & 70 & $1.1(-4.2 ; 6.5)$ & 0.674 \\
\hline
\end{tabular}

${ }^{\text {aP }}$ Primary outcome.

becondary outcome.

'Exploratory outcome.

the primary outcome analysis. The lack of contact between the researchers and participants between 1 year and 3 years is likely to have reduced engagement, resulting in one-third of participants not being followed-up. Weight loss at 1 year for the participants who we obtained follow-up measurements at 3 years was greater (TDR $-11 \mathrm{~kg}$ SD 9.5, UC $-3.2 \mathrm{~kg}$ SD 7.3) than for the people who we were unable to obtain measurements from at the extended follow-up (TDR -9.2 kg SD 10.6; UC $-2.6 \mathrm{~kg}$, SD 5.9). This suggests that the present results may reflect a more optimistic estimate of absolute weight loss in both groups.

We dealt with the missing data by using mixed models and extensive sensitivity analyses which consistently showed that the TDR group had greater weight loss than the nurse-delivered programme at 3 years. The trial was not powered to detect differences in cardiovascular risk factors. As such, the sample was insufficient to estimate the effect on blood pressure, and the confidence intervals range from no effect to a large effect. That said, the point estimate of the difference in blood pressure between arms is as would be expected from a prior meta-analysis, a reduction of $\sim 3 \mathrm{mmHg}$, given the $3.6 \mathrm{~kg}$ difference in weight [19].

\section{Comparisons with other studies}

A systematic review comparing TDRs with behavioural weight loss programmes reported that only four out of 12 studies followed people for 3 or more years [9]. A meta-analysis of these studies reporting longer-term follow-up showed a weight loss difference of only $-1.3 \mathrm{~kg}(-2.9$ to 0.2 ), somewhat less than the $-3.3 \mathrm{~kg}$ $(-5.2$ to -1.5$)$ seen here. Since this review, the DiRECT trial which tested a TDR programme for people with type 2 diabetes, has reported weight change at 2 years [20]. The rate of weight regain appears comparable to that we report here (Fig. S3). This is notable because the behavioural support in the DiRECT trial continued throughout the 2-year follow-up whereas in DROPLET it ceased after 6 months. Clarifying whether there is a benefit of ongoing support for the maintenance of weight-loss is important because it has implications for the cost of providing the intervention.

Weight regain in all trials of TDR is noticeably greater in absolute terms in the TDR programme than in the comparator group who lose less weight more slowly. This has prompted concerns that rapid weight loss is associated with rapid weight regain post-intervention. However, an experimental study which tested this hypothesis found no difference in the rate of weight regain following $15 \%$ weight loss achieved either with a TDR programme or a standard behavioural programme with more modest energy restriction over a longer period of time [21]. Instead it appears that the rate of weight regain reflects the absolute magnitude of weight loss, and our findings on weight regain are consistent with the extended follow up seen in trials of intensive weight loss interventions, which did not use a TDR approach, such as LookAHEAD [22]. Despite greater weight regain, the benefit of the greater initial weight loss with TDR persists to at least 3 years and a quarter of people who received a TDR programme were at least $10 \%$ lighter after 3 years, more than twice that in the UC group.

At follow-up, 7 in 10 participants in both groups reported currently trying to lose weight, similar to the proportion of people 
with obesity who report attempting to diet in the nationally representative Health Survey for England [23]. However in the trial, about half of the people who reported dieting were using a formal weight loss programme, which is associated with greater weight loss success than self-guided weight loss attempts. Our data suggest that people from more deprived neighbourhoods may have benefited relatively less at 3 years from the TDR than those from less deprived neighbourhoods, though the reasons for this are unclear. People from the most deprived neighbourhoods were no less likely to report being in formal weight loss programme at 3 years, but we cannot exclude the possibility that there were differences in attendance at weight-loss programmes between programme end and follow up especially since these programmes usually require self-funding $[24,25]$. Overall these data suggest that the pattern of weight change reported here, and in most trials, of weight loss followed by weight regain, is likely to conceal a series of efforts by individuals at different times, with weight fluctuating on a shorter cycle time than that described by the mean weight change trajectory.

No previous trials of TDR programmes in routine care settings other than DiRECT have reported on metabolic or cardiovascular effects beyond 1 year. One previous experimental study that examined weight change 3 years after TDR also found that the rate of regain of body weight and body fat regain was greater in the TDR group compared with the control. However, as in the present study, the greater initial losses in the TDR group were such that there was a persistent advantage to the TDR intervention over control at 3 years [26]. In the present study, despite the greater weight loss following TDR, our study produced equivocal evidence on the longer-term benefits on blood pressure, but no evidence of greater improvements in glycaemic control or overall cardiovascular risk compared with UC at 3 years. These results are compatible with trials of other weight-loss interventions, which seem to suggest that partial weight regain removes more of the effect of weight loss on cardiometabolic parameters than might be expected simply from the change in weight [27]. This may reflect disproportionate changes in risk factors during periods of weight loss or gain.

\section{Implications of this research}

This trial shows that offering a short TDR programme leads to longterm weight loss. We have previously reported that a TDR programme delivered by lay counsellors in the community is a cost effective treatment for weight loss [11]. The weight regain trajectories following TDR assumed in the cost effectiveness modelling were similar to those reported here, although weight regain in the UC group was somewhat slower than previously predicted such that the incremental cost effectiveness ratio would be lower. The DiRECT trial provides some evidence that offering a TDR improves glycaemic control in people with type 2 diabetes which is sufficient for at least a third of patients to be in remission at 2 years [20]. However, although underpowered to detect small effects, in the unselected population with obesity in DROPLET, there was little evidence that the TDR intervention led to greater improvements in cardiometabolic risk at 3 years. In practice it is these changes in risk factors for disease that are crucial to determining the cost effectiveness of interventions to treat obesity. Healthcare commissioners will need to carefully consider whether the routine provision of TDR programmes, which are substantially more costly than standard behavioural programmes, is warranted for people with obesity, but without pre-existing comorbidity.

\section{CONCLUSION}

A short TDR programme shows long term sustained improvements in weight in a general population of people with obesity. However, the lack of additional benefits on cardiometabolic risk suggests that commissioning these services for the treatment of obesity in the general population may not prove cost effective.

\section{DATA AVAILABILITY}

De-identified participant level data are available on request from the University of Oxford, Nuffield Department of Primary Care Data Access Committee for researchers who meet the criteria for access to confidential data: information.guardian@phc.ox. ac.uk.

\section{REFERENCES}

1. Astbury NM, Aveyard P, Nickless A, Hood K, Corfield K, Lowe R, et al. Doctor Referral of Overweight People to Low Energy total diet replacement Treatment (DROPLET): pragmatic randomised controlled trial. BMJ. 2018:362:k3760.

2. Lean ME, Leslie WS, Barnes AC, Brosnahan N, Thom G, McCombie L, et al. Primary care-led weight management for remission of type 2 diabetes (DiRECT): an openlabel, cluster-randomised trial. Lancet. 2017;391:541-51.

3. Taheri S, Zaghloul H, Chagoury O, Elhadad S, Ahmed SH, El Khatib N, et al. Effect of intensive lifestyle intervention on bodyweight and glycaemia in early type 2 diabetes (DIADEM-I): an open-label, parallel-group, randomised controlled trial. Lancet Diabetes Endocrinol. 2020;8:477-89.

4. Ard JD, Lewis KH, Rothberg A, Auriemma A, Coburn SL, Cohen SS, et al. Effectiveness of a Total Meal Replacement Program (OPTIFAST Program) on Weight Loss: results from the OPTIWIN Study. Obesity (Silver Spring). 2019;27:22-9.

5. Christensen $P$, Meinert Larsen $T$, Westerterp-Plantenga $M$, Macdonald I, Martinez JA, Handjiev S, et al. Men and women respond differently to rapid weight loss: Metabolic outcomes of a multi-centre intervention study after a low-energy diet in 2500 overweight, individuals with pre-diabetes (PREVIEW). Diabetes Obes Metab. 2018;20:2840-51.

6. Brown A, Dornhorst A, McGowan B, Omar O, Leeds AR, Taheri S, et al. Low-energy total diet replacement intervention in patients with type 2 diabetes mellitus and obesity treated with insulin: a randomized trial. BMJ Open Diabetes Res Care. 2020;8.:e001012.

7. NHS. The NHS Long Term Plan 2019 [https://www.longtermplan.nhs.uk/wpcontent/uploads/2019/08/nhs-long-term-plan-version-1.2.pdf.]

8. NHS. Low calorie diets to treat obesity and Type 2 diabetes 2020 [https://www. england.nhs.uk/diabetes/treatment-care/low-calorie-diets/]

9. Parretti HM, Jebb SA, Johns DJ, Lewis AL, Christian-Brown AM, Aveyard P. Clinical effectiveness of very-low-energy diets in the management of weight loss: a systematic review and meta-analysis of randomized controlled trials. Obesity Rev. 2016;17:225-34.

10. Fogelholm M, Larsen TM, Westerterp-Plantenga M, Macdonald I, Martinez JA, Boyadjieva $\mathrm{N}$, et al. PREVIEW: prevention of Diabetes through Lifestyle Intervention and Population Studies in Europe and around the World. Design, Methods, and Baseline Participant Description of an Adult Cohort Enrolled into a Three-Year Randomised Clinical Trial. Nutrients. 2017;9:2072-6643.

11. Kent S, Aveyard P, Astbury N, Mihaylova B, Jebb SA. Is Doctor Referral to a LowEnergy Total Diet Replacement Program Cost-Effective for the Routine Treatment of Obesity? Obesity (Silver Spring). 2019;27:391-8.

12. National Institute for Health Care Excellence (NICE). Weight management: lifestyle services for overweight or obese adults [PH53]. London: National Institute of Health Care Excellence; 2014.

13. Jebb SA, Astbury NM, Tearne S, Nickless A, Aveyard P. Doctor Referral of Overweight People to a Low-Energy Treatment (DROPLET) in primary care using total diet replacement products: a protocol for a randomised controlled trial. BMJ Open. 2017;7:e016709.

14. NHLBI Obesity Education Initiative. The practical guide: identification, evaluation, and treatment of overweight and obesity in adults. Maryland: National Institutes of Health, NIH Publication Number (00-4084) 2000.

15. Niero M, Martin M, Finger T, Lucas R, Mear I, Wild D, et al. A new approach to multicultural item generation in the development of two obesity-specific measures: the Obesity and Weight Loss Quality of Life (OWLQOL) questionnaire and the Weight-Related Symptom Measure (WRSM). Clin Ther. 2002;24:690-700.

16. EuroQol G. EuroQol-a new facility for the measurement of health-related quality of life. Health Policy. 1990;16:199-208.

17. Levy JC, Matthews DR, Hermans MP. Correct homeostasis model assessment (HOMA) evaluation uses the computer program. Diabetes Care. 1998;21:2191-2.

18. Department for Communities and Local Government. The English indicies of Deprivation 2015 [https://www.gov.uk/government/statistics/english-indices-ofdeprivation-2015.]

19. Neter JE, Stam BE, Kok FJ, Grobbee DE, Geleijnse JM. Influence of weight reduction on blood pressure: a meta-analysis of randomized controlled trials. Hypertension. 2003;42:878-84.

20. Lean MEJ, Leslie WS, Barnes AC, Brosnahan N, Thom G, McCombie L, et al. Durability of a primary care-led weight-management intervention for remission of type 2 diabetes: 2-year results of the DiRECT open-label, cluster-randomised trial. Lancet Diabetes Endocrinol. 2019;7:344-55. 
21. Purcell K, Sumithran P, Prendergast LA, Bouniu CJ, Delbridge E, Proietto J. The effect of rate of weight loss on long-term weight management: a randomised controlled trial. Lancet Diabetes Endocrinol. 2014;2:954-62.

22. The Look Ahead Research GC-oA. Eight-year weight losses with an intensive lifestyle intervention: the look AHEAD study. Obesity. 2014;22:5-13.

23. Piernas $C$, Aveyard $P$, Jebb SA. Recent trends in weight loss attempts: repeated cross-sectional analyses from the health survey for England. Int J Obes (Lond). 2016;40:1754-59.

24. Hartmann-Boyce J, Jebb S, Fletcher B, Aveyard P. Self-help for weight loss in overweight and obese adults: systematic review and meta-analysis. Am J Public Health. 2015;105:e43-57.

25. Hartmann-Boyce J, Johns DJ, Jebb SA, Summerbell C, Aveyard P. Behavioural Weight Management Review G. Behavioural weight management programmes for adults assessed by trials conducted in everyday contexts: systematic review and meta-analysis. Obesity Rev. 2014;15:920-32.

26. Seimon RV, Wild-Taylor AL, McClintock S, Harper C, Gibson AA, Johnson NA, et al. 3-Year effect of weight loss via severe versus moderate energy restriction on body composition among postmenopausal women with obesity - the TEMPO Diet Trial. Heliyon. 2020;6:e04007.

27. Kroeger CM, Hoddy KK, Varady KA. Impact of Weight Regain on Metabolic Disease Risk: a Review of Human Trials. J Obesity. 2014:614519.

\section{ACKNOWLEDGEMENTS}

We thank the participants and the staff at the primary care practices who contributed to this study and staff at the Oxford Primary Care and Vaccines Clinical Trials Unit for support in running this trial, particularly Sadie Kelly and David Watt for acting as data managers. We are also grateful to the Thames Valley Clinical Research Network Research Nurses: Sarah Wytrykowski, Debbie Kelley, Diane Lonsdale, Lydia Owen and Heather Edwards assisted in the collection of measurements for this study.

\section{AUTHOR CONTRIBUTIONS}

NMA, SAJ and PA conceived the idea and obtained funding. NMA and RE collected the data. FG, MS and JM analysed the data. NMA wrote the first draft of the paper in collaboration with SAJ and PA. All authors contributed to the interpretation of the data and critical review of the paper. NMA affirms that the paper is an honest, accurate, and transparent account of the study being reported; that no important aspects of the study have been omitted; and that any discrepancies from the study as planned (and, if relevant, registered) have been explained.

\section{FUNDING}

This study was funded by National Institute of Health Research Applied Research Collaboration at Oxford Health NHS Foundation Trust. SAJ, PA, and NA are also supported by the Oxford NIHR Biomedical Research Centre and SAJ and PA are NIHR senior investigators. The study was conducted and analysed in collaboration with the Oxford Primary Care and Vaccines clinical trials unit. The funders had no role in study design, data collection, data analysis, data interpretation, or writing of the report. The views are those expressed by the authors and not necessarily those of the NHS, NIHR, or Department of Health. The University of Oxford holds the relevant clinical trials insurance policy for this study and acted as the study sponsor.

\section{COMPETING INTERESTS}

All authors have completed the Unified Competing Interest form (available on request from the corresponding author) and declare: NMA, SAJ and PA were investigators on the DROPLET clinical trial which was funded by an investigatorinitiated research grant from Cambridge Weight Plan UK Ltd to the University of Oxford. SAJ and NMA are investigators on a trial which digital weight loss interventions have been provided by Slimming World and Rosemary Conley to the participants of the trial free of charge. PA did half a day's consultancy for Weight Watchers and spoke at a symposium funded by Novo Nordisk. SAJ attended a 1-day meeting on digital health interventions, organised by Oviva UK Ltd, in which expenses were paid to the University of Oxford. None of these activities have led to personal payments. SAJ and PA are members of NHS England advisory boards relating to weight-loss interventions for the prevention and treatment of type 2 diabetes and obesity. RE, FG, MS and JM have no competing interests to declare.

\section{ADDITIONAL INFORMATION}

Supplementary information The online version contains supplementary material available at https://doi.org/10.1038/s41366-021-00915-1.

Correspondence and requests for materials should be addressed to N.M.A.

Reprints and permission information is available at http://www.nature.com/ reprints

Publisher's note Springer Nature remains neutral with regard to jurisdictional claims in published maps and institutional affiliations.

Open Access This article is licensed under a Creative Commons Attribution 4.0 International License, which permits use, sharing, adaptation, distribution and reproduction in any medium or format, as long as you give appropriate credit to the original author(s) and the source, provide a link to the Creative Commons license, and indicate if changes were made. The images or other third party material in this article are included in the article's Creative Commons license, unless indicated otherwise in a credit line to the material. If material is not included in the article's Creative Commons license and your intended use is not permitted by statutory regulation or exceeds the permitted use, you will need to obtain permission directly from the copyright holder. To view a copy of this license, visit http://creativecommons. org/licenses/by/4.0/

(c) The Author(s) 2021 